Supporting Information

\title{
Bimodal Nanofiber and Microfiber Nonwovens by Melt-Blowing Immiscible Ternary Polymer Blends
}

Kailong Jin, Sarah Eyer, William Dean, David Kitto, Frank S. Bates, and Christopher J. Ellison* Department of Chemical Engineering and Materials Science, University of Minnesota, Minneapolis, MN 55455, United States

*To whom correspondence should be addressed: cellison@umn.edu

\section{Materials and Methods.}

Materials. Commercial polymers, including polyethylene (PE; linear low density), nylon 6 (Nylon), polystyrene (PS; bimodal), and poly(ethylene oxide) (PEO), were purchased from Sigma-Aldrich and used as received. To provide more details, the Sigma-Aldrich product numbers for linear low density PE, Nylon 6, PS, and PEO used in this study are 428108, 181110, 331651, and 81300, respectively. The linear low density PE used in this study has a density of $0.9215-0.9255 \mathrm{~g} / \mathrm{ml}$, a melt flow index of $100 \mathrm{~g} / 10 \mathrm{~min}\left(190^{\circ} \mathrm{C} / 2.16 \mathrm{~kg}\right)$, and a melting temperature of $\sim 125{ }^{\circ} \mathrm{C}$ (measured by the peak temperature of the endothermic peak on DSC thermogram in Figure S1). The bimodal PS used in this study has a weight average molecular weight $\left(M_{\mathrm{w}}\right) \sim 35 \mathrm{~kg} / \mathrm{mol}$ and is comprised of $\sim 30$ and $\sim 70 \mathrm{wt} \%$ of unimodal PS with $M_{\mathrm{w}}$ of 115 and $2.5 \mathrm{~kg} / \mathrm{mol}$, respectively. The PEO used in this study had a nominal number average molecular weight, $M_{\mathrm{n}}=20 \mathrm{~kg} / \mathrm{mol}$. Solvents, including tetrahydrofuran ( $\geq 99.6 \%$; Fisher), formic acid (88\%; Macron Fine Chemicals ${ }^{\mathrm{TM}}$, Avantor), and toluene ( $\geq 99.5 \%$; Fisher), were used as received.

Preparation of ternary polymer blends. PS, PE, Nylon, and PEO were dried overnight in a 
vacuum oven at $80{ }^{\circ} \mathrm{C}$ to remove moisture before melt blending in a recirculating, conical twinscrew batch mixer (DSM Xplore, $5 \mathrm{~mL}$ capacity) operated at $100 \mathrm{rpm}$ with a steady dry nitrogen purge. Ternary immiscible PS/PE/Nylon blends (50/5/45 wt $\%, 50 / 15 / 35 \mathrm{wt} \%$, and 50/25/25 wt $\%$ PS/PE/Nylon blends) were prepared by first melt mixing appropriate amounts of PS and PE at $240{ }^{\circ} \mathrm{C}$ for $5 \mathrm{~min}$, followed by the addition of Nylon and mixing for another $3 \mathrm{~min}$. The resulting mixture was extruded through a $2.5 \mathrm{~mm}$ diameter die and air cooled. As an example, a batch of 50/5/45 wt $\%$ PS/PE/Nylon blend contains $2 \mathrm{~g}$ of PS, $0.2 \mathrm{~g}$ of PE, and $1.8 \mathrm{~g}$ of Nylon. The 60/5/35 wt \% PEO/PE/Nylon blend was prepared in a similar manner. The compositions for both $\mathrm{PS} / \mathrm{PE} / \mathrm{Nylon}$ and PEO/PE/Nylon ternary blends were chosen to obtain desired blend morphologies, i.e., with the two minority blend components being randomly dispersed as isolated, bimodally sized particles within the continuous matrix. For PS/PE/Nylon ternary blends, the PS composition was fixed at $50 \mathrm{wt} \%$ to ensure that PS serves as a continuous matrix. For PEO/PE/Nylon ternary blends, the PEO composition was fixed at $60 \mathrm{wt} \%$ to ensure that PEO serves as a continuous matrix. In both cases, both PE and Nylon compositions were varied, accordingly.

Melt blowing. To produce nonwoven fibers, the ternary blends were loaded into a custom-built lab-scale melt blowing apparatus, which contained a homemade melt blowing die and fiber collector fit to a commercial capillary rheometer (Goettfert ${ }^{\mathrm{TM}}$ Rheo-Tester 1500). (See Refs. 1-3 for more details regarding the set-up of our lab-scale melt blowing apparatus.) The capillary rheometer was used to heat the polymer to the melt blowing temperature and extrude the polymer through a single-hole melt blowing orifice die with an diameter of $200 \mu \mathrm{m}$ at a controlled polymer flow rate of $0.2 \mathrm{~g} /(\mathrm{min}$ hole). Melt blowing was carried out $5 \mathrm{~min}$ after the sample reached the melt blowing temperature (required about 5 min to reach temperature). The 
air flow rate was 3.8 standard cubic feet per minute (SCFM) and the air pressure at the die exit

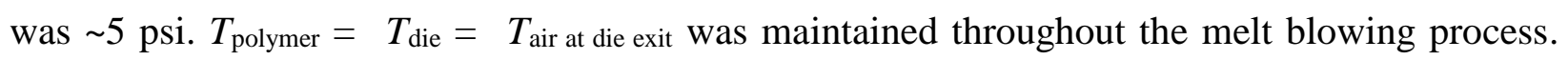
Melt blown fibers were collected using a stationary collector consisting of a stainless-steel screen.

Typical melt blowing conditions/parameters used for melt blown nonwoven production are listed here: (1) A key property for melt blowing is melt viscosity, which can be measured by the complex viscosity $\left(\eta^{*}\right)$ from dynamic rheological measurements. According to Figure S2, $\eta^{*}(\mathrm{PS})=\sim 1.5 \mathrm{~Pa} \mathrm{~s}, \eta^{*}(\mathrm{PE})=\sim 45 \mathrm{~Pa} \mathrm{~s} ; \eta^{*}($ Nylon $)=\sim 300 \mathrm{~Pa}$ at $240{ }^{\circ} \mathrm{C}$ and a frequency of 2 $\mathrm{rad} / \mathrm{s}$; similarly, $\eta^{*}(\mathrm{PEO})=\sim 2 \mathrm{~Pa} \mathrm{~s}, \eta^{*}(\mathrm{PE})=\sim 50 \mathrm{~Pa} \mathrm{~s} ; \eta^{*}(\mathrm{Nylon})=\sim 650 \mathrm{~Pa}$ at $230{ }^{\circ} \mathrm{C}$ and a frequency of $2 \mathrm{rad} / \mathrm{s}$; (2) The distance from the melting blowing orifice and the collector is $\sim 1 \mathrm{~m}$. The velocity of the air at the die exit (greater than fiber velocity) is typically $\sim 100-300 \mathrm{~m} / \mathrm{s}$, resulting in an estimated fiber flight time of 10s of milliseconds. (3) The orifice and collector temperatures are melt blowing temperature $\left(240\right.$ or $230{ }^{\circ} \mathrm{C}$ in this study) and ambient temperature, respectively.

Selective extraction of blend components. (a) Selective extraction of PS: To remove PS, the sample (bulk blends or fibers) was soaked in tetrahydrofuran at $50{ }^{\circ} \mathrm{C}$ for $3 \mathrm{hr}$. Under these conditions, only PS can be dissolved but PE and Nylon cannot. The solution was then separated from the sample, and more fresh tetrahydrofuran was added afterwards. This procedure was repeated five times before drying the insoluble fractions at $50{ }^{\circ} \mathrm{C}$ for $12 \mathrm{hr}$. By comparing the weights of the sample before and after solvent extraction, the amount of PS being removed was quantified. (b) Selective extraction of Nylon: After PS removal, the sample was soaked in formic acid at room temperature (RT) for $3 \mathrm{hr}$. Under these conditions, only Nylon can be dissolved but PS and PE cannot. The same washing protocol was repeated five times to ensure 
the complete removal of Nylon. The weights of the sample before and after Nylon extraction were also quantified. (c) Selective extraction of PS and PE: To remove PS and PE simultaneously, the sample was soaked in toluene at $110^{\circ} \mathrm{C}$ for $3 \mathrm{hr}$. Under these conditions, PS and PE can be dissolved but Nylon cannot. The same washing protocol was repeated five times to ensure the complete removal of both PS and PE. The weights of the sample before and after PS and PE removal were characterized.

Differential scanning calorimetry (DSC). Differential scanning calorimetry was performed with a Mettler Toledo DSC 1 calorimetry instrument. Approximately $5 \mathrm{mg}$ of sample was loaded into aluminum pans for each DSC run. Materials were heated to $250{ }^{\circ} \mathrm{C}$ (or $140{ }^{\circ} \mathrm{C}$ for PS) to erase thermal history, cooled to $25{ }^{\circ} \mathrm{C}$ at $20{ }^{\circ} \mathrm{C} / \mathrm{min}$, and then heated to $250{ }^{\circ} \mathrm{C}$ (or $140{ }^{\circ} \mathrm{C}$ for PS) at $10{ }^{\circ} \mathrm{C} / \mathrm{min}$. Glass transition $\left(T_{\mathrm{g}, 1 / 2 \Delta \mathrm{Cp}}\right.$ from DSC) and melting temperatures were determined from the second heating ramp.

Fourier transform infrared spectroscopy (FTIR). FTIR measurements were performed on an attenuated total reflection Fourier transform infrared spectroscopy (ATR-FTIR; Nicolett 6700, Thermo Scientific). All samples were scanned at a resolution of $2 \mathrm{~cm}^{-1}$, and 64 scans were collected in the range of $4000-600 \mathrm{~cm}^{-1}$.

Rheological measurements. Rheological properties were measured with an ARES rheometer (TA Instruments) equipped with a $25 \mathrm{~mm}$ parallel-plate fixture. All experiments were performed in the linear viscoelastic region, which was determined by dynamic strain sweeps. Isothermal dynamic frequency sweep measurements were conducted between 0.1 and $100 \mathrm{rad} / \mathrm{s}$ to measure elastic modulus $\left(G^{\prime}\right)$, viscous modulus $\left(G^{\prime \prime}\right)$, and complex viscosity $\left(\eta^{*}\right)$ at either 230 or $240{ }^{\circ} \mathrm{C}$.

Scanning electron microscope (SEM). (a) Blend morphology characterization: Polymer blends were freeze-fractured in liquid nitrogen then coated with about $5 \mathrm{~nm}$ of iridium (ACE600 
Coater) for morphological characterization by scanning electron microscope (SEM; Hitachi S4700 SEM). The blend components were selectively removed to expose PE and Nylon droplets, respectively. The size distribution of PE and Nylon particles was obtained by measuring 300 droplets in several SEM images using ImageJ software. (b) Fiber morphology characterization: A SEM sample was cut with scissors from the melt blown fiber mats and coated with $\sim 5 \mathrm{~nm}$ of iridium using an ACE600 Coater. For each fiber mat, 10-20 SEM micrographs were taken (in regions away from the cutting edges) and $\sim 300$ fiber diameter measurements were made using ImageJ software. (c) Determination of geometric average $\left(d_{a v}\right)$ values: The Origin Lab was employed to fit a lognormal distribution function (Equation S1) to the particle size and fiber diameter data. The $d_{\mathrm{av}}$ and standard deviation $(\sigma)$ of the fiber diameter distribution were extracted from the log-normal fitting. The geometric standard deviation (GSD) and coefficient of variation (CV) were calculated from the log-normal fitting results using Equations S2 and S3, respectively. ${ }^{3}$

$$
\begin{aligned}
& f(d)=\frac{1}{d \cdot \sigma \sqrt{2 \pi}} \exp \left[-\frac{1}{2 \sigma^{2}}\left\{\ln \left(\frac{d}{d_{a v}}\right)\right\}^{2}\right] \\
& G S D=\exp (\sigma) \\
& C V=\sqrt{\exp \left(\sigma^{2}\right)-1} \times 100 \%
\end{aligned}
$$




\section{Supporting Characterization and Results.}

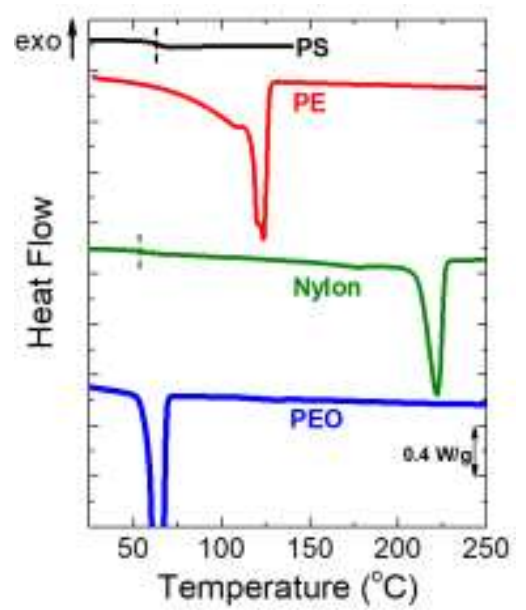

Figure S1. DSC thermograms of neat PS, PE, Nylon, and PEO. All thermograms were obtained from the second heating ramp at $10{ }^{\circ} \mathrm{C} / \mathrm{min}$ after annealing at $250{ }^{\circ} \mathrm{C}\left(140{ }^{\circ} \mathrm{C}\right.$ for PS $)$ for $5 \mathrm{~min}$, followed by quenching to $\mathrm{RT}$ at $20^{\circ} \mathrm{C} / \mathrm{min}$.
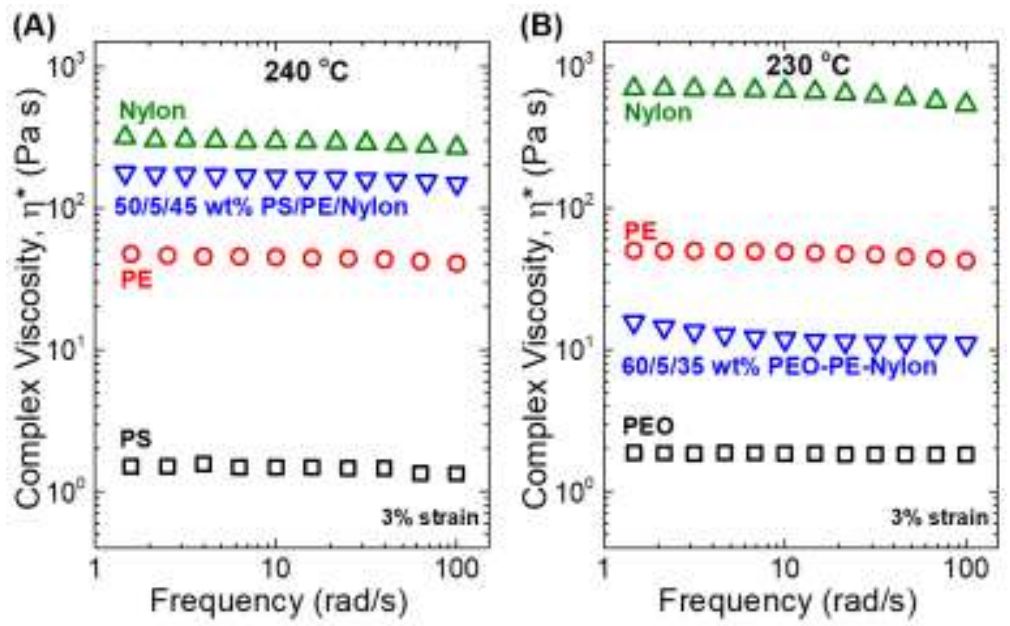

Figure S2. $\eta^{*}$ versus frequency for (A) neat PS, PE, Nylon, and 50/5/45 wt\% PS/PE/Nylon blend at $240{ }^{\circ} \mathrm{C}$ and (B) PEO, PE, Nylon, and 60/5/35 wt\% PEO/PE/Nylon blend at $230{ }^{\circ} \mathrm{C}$. All samples were preannealed for $5 \mathrm{~min}$ at the indicated temperatures before rheological measurements in the linear viscoelastic region. 


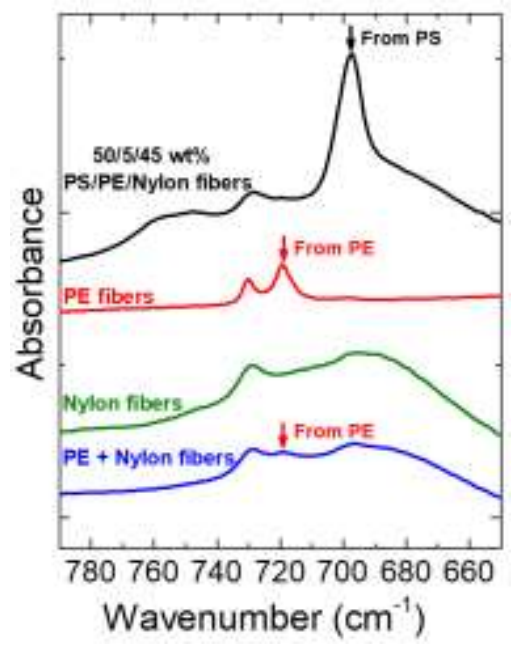

Figure S3. FTIR spectra from 650 to $790 \mathrm{~cm}^{-1}$ for 50/5/45 wt $\%$ PS/PE/Nylon fibers, extracted PE fibers, extracted Nylon fibers, and extracted PE + Nylon fibers.

(A) 50/15/35 wt \% PS/PE/Nylon

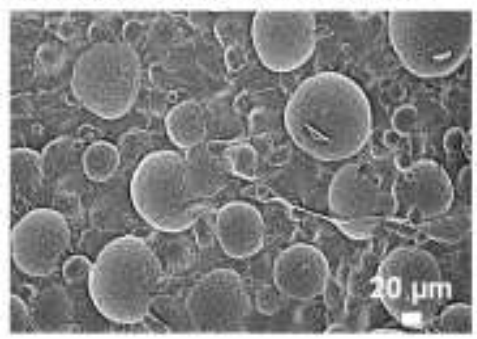

(B) $50 / 25 / 25 w t \% \mathrm{PS} / \mathrm{PE} / \mathrm{Nylon}$

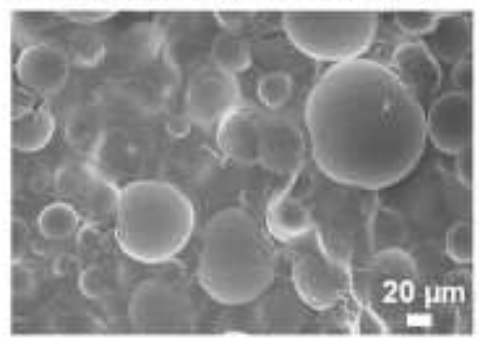

Figure S4. Representative SEM images of (A) 50/15/35 wt\% and (B) 50/25/25 wt\% PS/PE/Nylon blends. 
(A) PE fibers

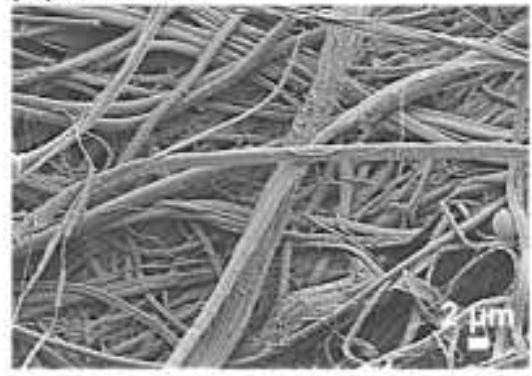

(C) Nylon fibers

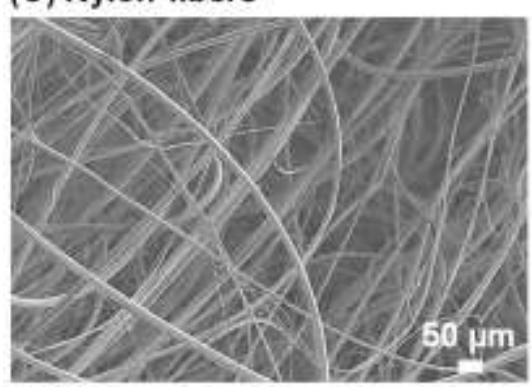

(B)

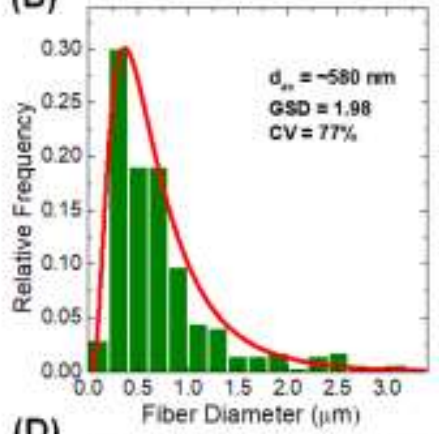

(D)

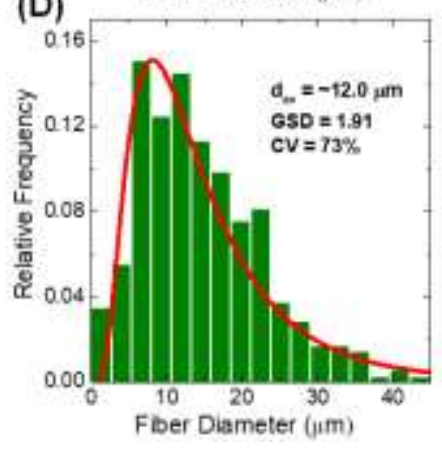

Figure S5. Representative SEM images of (A) PE fibers and (C) Nylon fibers extracted from melt blown 50/15/35 wt\% PS/PE/Nylon fibers. Statistical analysis of the fiber diameters in (A) and (C) are provided in (B) and (D), respectively. 
(A) PE fibers

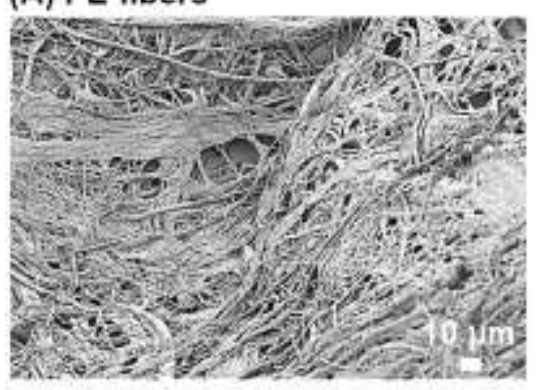

(C) Nylon fibers

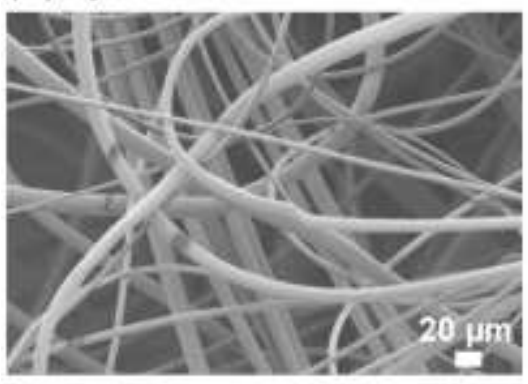

(B)

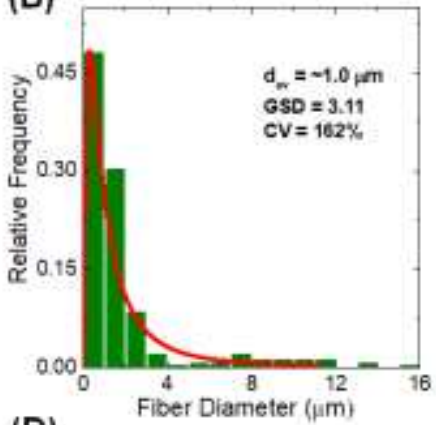

(D)

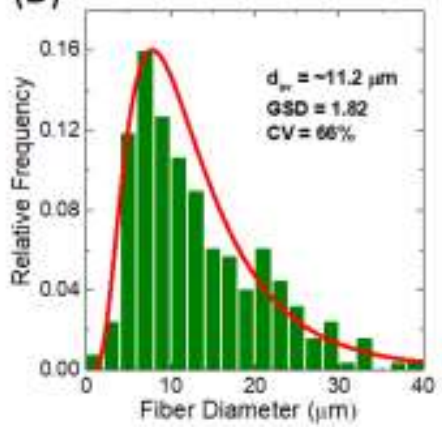

Figure S6. Representative SEM images of (A) PE fibers and (C) Nylon fibers extracted from melt blown 50/25/25 wt\% PS/PE/Nylon fibers. Statistical analysis of the fiber diameters in (A) and (C) are provided in (B) and (D), respectively. 

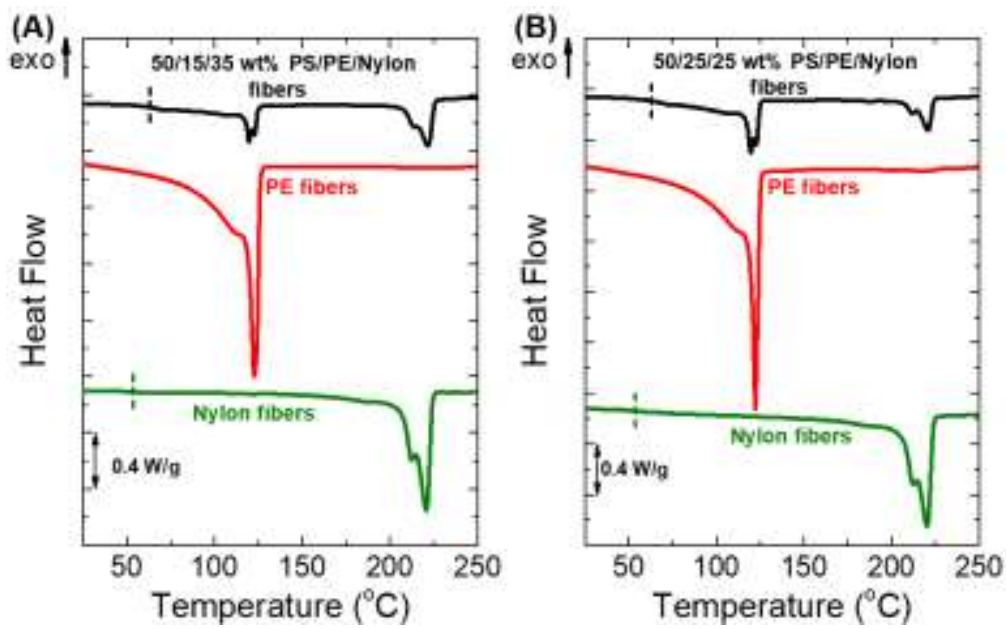

Figure S7. (A) DSC thermograms for 50/15/35 wt\% PS/PE/Nylon fibers, extracted PE fibers, and extracted Nylon fibers and (B) 50/25/25 wt\% PS/PE/Nylon fibers, extracted PE fibers, and extracted Nylon fibers. All thermograms were obtained from the second heating ramp at $10{ }^{\circ} \mathrm{C} / \mathrm{min}$ after annealing at $250{ }^{\circ} \mathrm{C}$ for $5 \mathrm{~min}$, followed by quenching to RT at $20{ }^{\circ} \mathrm{C} / \mathrm{min}$.

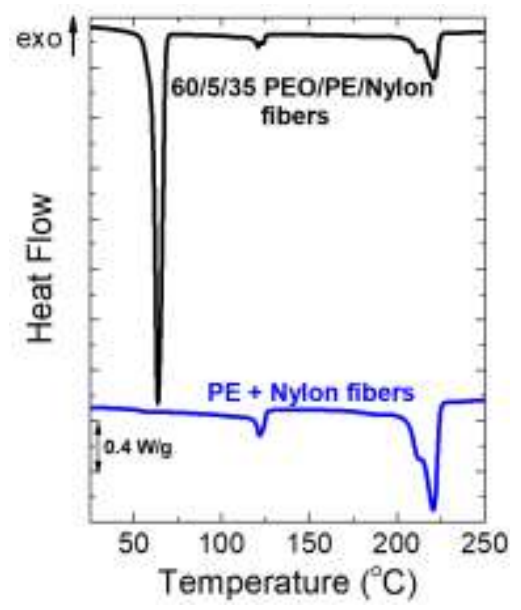

Figure S8. DSC thermograms for 60/5/35 wt\% PEO/PE/Nylon fibers and extracted PE + Nylon fibers. All thermograms were obtained from the second heating ramp at $10{ }^{\circ} \mathrm{C} / \mathrm{min}$ after annealing at $250{ }^{\circ} \mathrm{C}$ for $5 \mathrm{~min}$, followed by quenching to $\mathrm{RT}$ at $20^{\circ} \mathrm{C} / \mathrm{min}$. 
(A) PE fibers

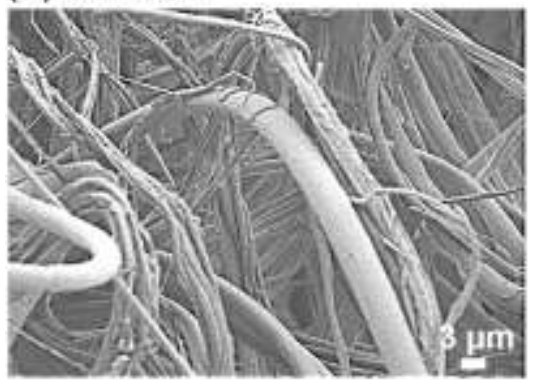

(C) Nylon fibers

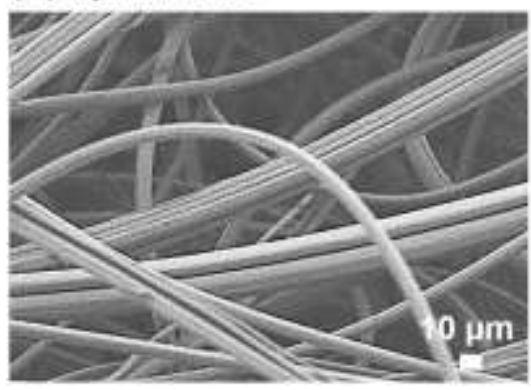

(B)

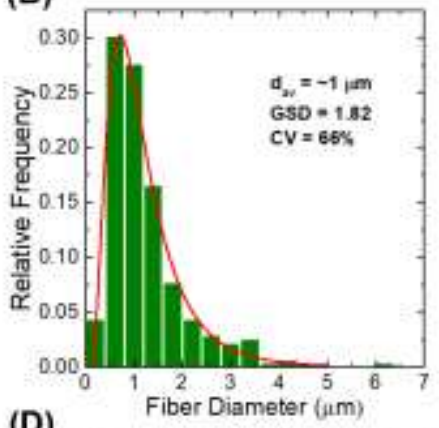

(D)

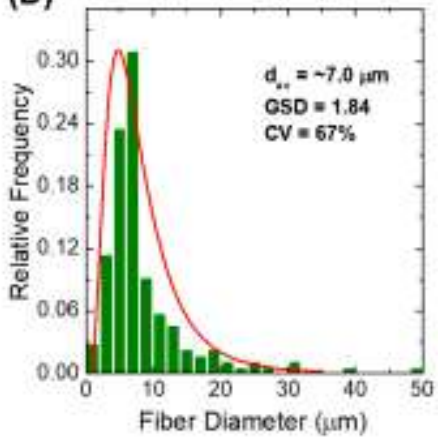

Figure S9. Representative SEM images of (A) PE fibers and (C) Nylon fibers extracted from melt blown 60/5/35 wt\% PEO/PE/Nylon fibers. Statistical analysis of the fiber diameters in (A) and (C) are provided in (B) and (D), respectively. The $d_{\mathrm{av}}$ values in the size distribution figures are the number-average diameters of $~ 300$ individual fibers.

Table S1. Interfacial tension values reported in the literature ${ }^{4-7}$ between different blend components.

\begin{tabular}{cc}
\hline $\begin{array}{c}\text { Blend } \\
\text { system }\end{array}$ & $\begin{array}{c}\text { Interfacial tension, } \gamma^{4-7} \\
(\mathrm{mN} / \mathrm{m})\end{array}$ \\
\hline PS/PE & $\sim 5$ \\
PS/Nylon & $\sim 7$ \\
PE/Nylon & $\sim 14$ \\
PEO/PE & $\sim 9$ \\
PEO/Nylon & $\sim 1$ \\
\hline
\end{tabular}




\section{References}

1. Zuo, F.; Tan, D. H.; Wang, Z.; Jeung, S.; Macosko, C. W.; Bates, F. S., Nanofibers from Melt Blown Fiber-in-Fiber Polymer Blends. ACS Macro Lett. 2013, 2 (4), 301-305.

2. Ellison, C. J.; Phatak, A.; Giles, D. W.; Macosko, C. W.; Bates, F. S., Melt Blown Nanofibers: Fiber Diameter Distributions and Onset of Fiber Breakup. Polymer 2007, 48 (11), 3306-3316.

3. Tan, D. H.; Zhou, C.; Ellison, C. J.; Kumar, S.; Macosko, C. W.; Bates, F. S., Meltblown Fibers: Influence of Viscosity and Elasticity on Diameter Distribution. J. Non-Newton. Fluid Mech. 2010, 165 (15), 892-900.

4. Wu, S., Calculation of Interfacial Tension in Polymer Systems. J. Polym. Sci. Polym. Symp. 1971, 34 (1), 19-30.

5. Xing, P.; Bousmina, M.; Rodrigue, D.; Kamal, M. R., Critical Experimental Comparison between Five Techniques for the Determination of Interfacial Tension in Polymer Blends: Model System of Polystyrene/Polyamide-6. Macromolecules 2000, 33 (21), 8020-8034.

6. Fortelny, I.; Lapcikova, M.; Mikesova, J.; Juza, J., The Effect of Polyethylene Addition on the Morphology of Polystyrene/Polyamide Blends. J. Polym. Sci. Polym. Phys. 2009, 47 (21), 2158-2170.

7. Roe, R.-J., Interfacial Tension Between Polymer Liquids. J. Colloid Interface Sci. 1969, $31(2), 228-235$. 